\title{
Global neonatal and perinatal mortality: a review and case study for the Loreto Province of Peru
}

\author{
This article was published in the following Dove Press journal: \\ Research and Reports in Neonatology \\ 27 October 2012 \\ Number of times this article has been viewed
}

\author{
Jamie B Warren' \\ William E Lambert ${ }^{2}$ \\ Rongwei $\mathrm{Fu}^{2}$ \\ JoDee MAnderson' \\ Alison B Edelman ${ }^{3}$ \\ 'Department of Pediatrics, \\ ${ }^{2}$ Department of Public Health \\ and Preventive Medicine, ${ }^{3}$ Department \\ of Obstetrics and Gynecology, \\ Oregon Health and Science University, \\ Portland, OR, USA
}

Background: Millennium Development Goal 4 calls for the reduction of the under-five mortality rate by two-thirds between 1990 and 2015. To reach this goal, neonatal mortality must be decreased. The lack of information on global neonatal and perinatal mortality impedes appropriate implementation of interventions, as vital registration systems are not available for the majority of the world's neonatal deaths. Verbal autopsy (VA) is a tool that has been used to determine cause of death. Recent studies have attempted to standardize and validate the use of this tool in resource-limited areas. The World Health Organization (WHO) International Standard VA Questionnaire was used to conduct a needs assessment in nine rural Peruvian villages. The goal was to determine the neonatal mortality rate (NMR), perinatal mortality rate (PMR), and causes of, and risk factors for, death in these villages.

Methods: Eligible women were interviewed using the WHO International Standard VA Questionnaire or a set of questions based on the WHO VA Questionnaire. NMR and PMR were calculated using a generalized estimating equation model. Three neonatologists independently reviewed VA records to provide cause of death determination. Reviewer agreement was assessed using percent agreement. Fisher's exact test was used to determine risk factors associated with death.

Results: The NMR was 31.4 per 1000 live births and the PMR was 49.7 per 1000 pregnancies. The main contributor to neonatal death was infection (43\%). Percent agreement among reviewers was $90.5 \%$ and $38.9 \%$ for cause of neonatal death and stillbirth, respectively. Risk factors for death were pregnancy with twins $(P=0.001)$, preterm delivery $(P=0.003)$, and cesarean section delivery $(P=0.049)$.

Conclusion: The WHO VA proved useful for NMR and PMR calculation, cause of death determination, and risk factor identification. Information gathered in this needs assessment will allow for the design and implementation of tailored interventions.

Keywords: neonatal mortality, perinatal mortality, verbal autopsy, needs assessment

\section{Introduction}

In September of 2000, heads of State and Government from 192 countries adopted the Millennium Development Goals, which have an overall focus on issues including poverty, education, gender equality, health, and environmental sustainability. ${ }^{1,2}$ Millennium Development Goal 4 (MDG4) calls for the reduction of the under-five mortality rate by two-thirds between 1990 and 2015. ${ }^{3}$ Strides are being made in this critical area of child health, as the under-five mortality rate has fallen from 88 per 1000 live births in 1990 to 57 per 1000 live births in 2010. ${ }^{4}$ However, the current rate of reduction must increase six-fold to reach MDG4. ${ }^{5}$ 
Many believe that MDG4 can be achieved through an expansion of interventions that target the most important causes of death. ${ }^{6}$ In 2008, four diseases of childhood pneumonia, diarrhea, malaria, and acquired immunodeficiency virus - accounted for $38 \%$ of deaths in children under the age of five worldwide (Figure 1). ${ }^{7}$ While progress has been made in these and other causes of under-five mortality, deaths that occur in the neonatal time period are decreasing at a much slower rate. In $2008,41 \%$ of deaths in children under the age of five occurred in neonates (Figure 1), a proportion that has increased from the $38.9 \%$ share of under-five deaths that occurred in neonates in $19700^{7,8}$ This increasing share of under-five childhood death that is occurring in the neonatal time period brings neonatal mortality to the forefront: to meet MDG4, neonatal mortality must be addressed. ${ }^{6}$ In fact, estimations state that prevention of $70 \%$ of neonatal deaths worldwide would decrease the under-five mortality rate by $25 \%{ }^{9}$

\section{Neonatal and perinatal death: a global perspective}

Of the over 130 million babies born every year worldwide, about 3.6 million of them will die before reaching 1 month of age. ${ }^{4,5}$ For every neonatal death, it is estimated that one stillbirth occurs; around one million of these are thought to be intrapartum stillbirths. ${ }^{10,11}$ These deaths stem from poor maternal health, inadequate prenatal care, inappropriate management of complications during labor and delivery, poor hygiene during delivery and just after birth, and lack of the

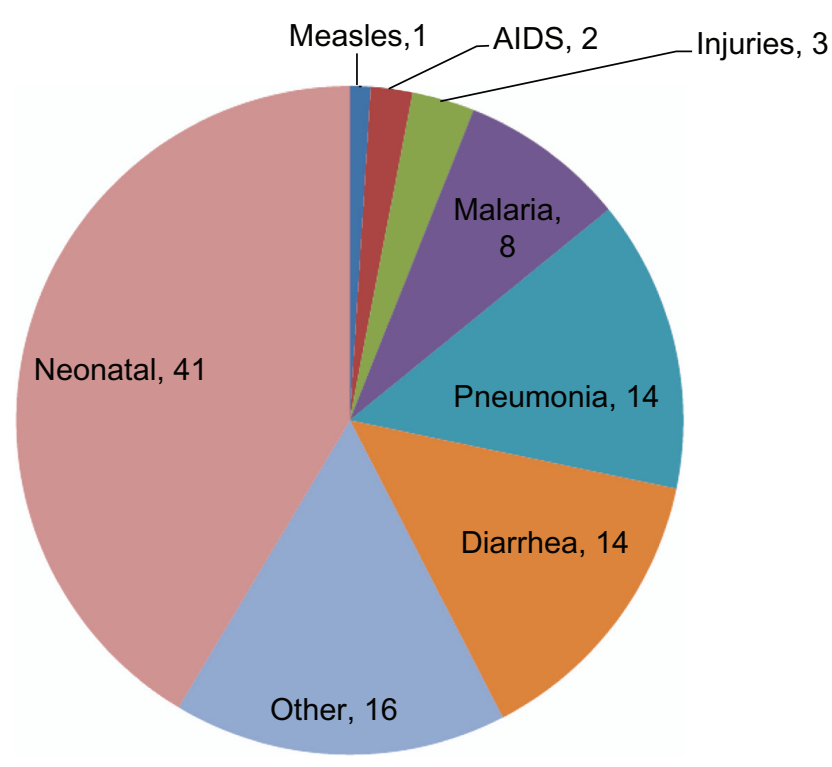

Figure I Causes of death among children under-five worldwide in 2008 (\%). ${ }^{7}$ Abbreviation: AIDS, acquired immunodeficiency syndrome. essential newborn care that is needed during the first days of life. ${ }^{12}$ Within the neonatal period, there is great variability in the daily risk of death. Mortality is highest in the initial 24 hours after birth, up to $50 \%$ die within the first 3 days of life, and about $75 \%$ of all neonatal deaths occur in the first week of life (early neonatal death).,13

Due to a lack of vital registration systems in many areas of the world, global estimates of frequency of direct causes of neonatal death exist only by means of statistical modeling. ${ }^{14}$ Based on estimates from 2008, the main causes of neonatal death and their estimates of occurrence are infection (29\%), preterm birth (29\%), birth asphyxia (23\%), and congenital anomalies (8\%). ${ }^{5,6}$ Causes of death can be correlated with timing of death. Neonatal death that occurs in the first week of life is often related to complications of birth asphyxia and prematurity, while deaths occurring after 1 week of life are commonly due to infectious causes. ${ }^{12,14}$ In a study of over 1000 neonatal deaths in rural India, $31 \%$ and $26 \%$ of deaths on the first day of life were due to birth asphyxia and preterm birth, respectively. ${ }^{15}$ For the remainder of the first week, $30 \%$ of deaths were due to preterm birth and $25 \%$ were due to sepsis and pneumonia. ${ }^{15}$ Infection accounted for $45 \%$ of deaths during the second week of life and $36 \%$ of deaths during weeks three and four. ${ }^{15}$

Risk factors have been identified from populationbased studies for all-cause neonatal and perinatal death. ${ }^{11}$ Prepregnancy risk factors include maternal age $<18$ years or $>35$ years, maternal height $<150 \mathrm{~cm}$ and weight $<47 \mathrm{~kg}$, primigravid status or parity greater than six, and poor obstetric history. ${ }^{11}$ Antenatal risk factors include multiple pregnancy; maternal anemia, jaundice, hypertensive disorders, diabetes, syphilis, malaria, or human immunodeficiency virus; preterm birth ( $<37$ weeks gestation); and postterm birth ( $>42$ weeks gestation). ${ }^{11}$ Intrapartum risk factors include obstructed labor, prolonged second stage of labor, passage of meconium, malpresentation (including breech), vaginal bleeding, maternal fever during labor, and prolonged rupture of membranes. ${ }^{11}$

The burden of neonatal mortality is rooted in inequality. In 2010, the neonatal mortality rate (NMR) in developing countries was 25 per 1000 live births, which is over eight times the NMR seen in industrialized countries (three per 1000 live births). ${ }^{4}$ In fact, $99 \%$ of neonatal deaths occur in developing countries. ${ }^{14}$ Two-thirds of these deaths occur in the World Health Organization (WHO) African and southeast Asian regions. ${ }^{14}$ The effect of inequality is seen within countries as well, as NMRs are highest in the poorest wealth quintile of many developing countries. ${ }^{5}$ 
Lack of access to skilled care is a major contributor to high NMRs in developing countries. In about half of the deliveries in developing countries, skilled care in the form of a birth attendant with formal education is unavailable. ${ }^{10}$ Nearly two-thirds of births that occur in developing countries and up to $90 \%$ of deliveries in the poorest wealth quintile of many developing countries occur in the home. ${ }^{9,10}$ Between 1995 and 2003, over 50\% of neonatal deaths came after a delivery that occurred in the home without the presence of a skilled birth attendant. ${ }^{14}$ Lack of access to care occurs before and extends beyond the time of delivery as well. Prenatal care is often unavailable, and most babies in developing countries are cared for at home without any formal medical care in the days immediately following delivery. ${ }^{13}$

\section{Neonatal and perinatal death: data collection and verbal autopsy (VA)}

The ability to study neonatal and perinatal mortality, as well as plan and implement programs to improve survival, is impeded by an overall lack of information. ${ }^{16}$ Countries with the highest NMRs often lack the vital registration systems that are necessary to record information about deaths. ${ }^{17}$ Death registration is reliable in only 72 of the world's $>190$ countries, and vital registration coverage is available for less than $3 \%$ of the world's neonatal deaths. ${ }^{1,11}$ Without vital registration systems, direct cause of death statistics for certain countries and regions are not available, resulting in the need for global estimates based on statistical modeling. ${ }^{14}$ In countries that do have vital registration systems, the high number of home deliveries that occur in resource-poor settings likely leads to high numbers of uncounted and uncharacterized deaths. ${ }^{18}$ High rates of stillbirth that occur during home deliveries, and the frequent misclassification of neonatal death as stillbirth in home deliveries, further contribute to issues surrounding vital registration. ${ }^{19}$

VA is one tool that is used to determine cause of death in places lacking vital registration systems. ${ }^{20} \mathrm{VA}$ consists of an interview with family members of the deceased using a structured questionnaire to elicit findings that can be used to determine cause of death. ${ }^{20}$ Despite known limitations of VA, including questions about validation and use for comparison between countries, this is currently the only practical tool available to monitor causes of death in developing countries without vital registration systems. ${ }^{20}$ Because the places lacking these vital registration systems are the very places targeted by recent global health initiatives, the need for standardized VA-derived mortality data has increased.
In 2007, the WHO published VA standards to be used in evaluation of such initiatives, as well as to provide a source of cause of death statistics to make disease burden estimates. ${ }^{20}$ These VA standards were the product of a 3-year effort by an expert group lead by the WHO. This expert group - consisting of researchers, data users, and other stakeholders - systematically reviewed, debated, and refined the currently available data and evidence from the most widely previously used and validated VA questionnaires. ${ }^{20}$ The results were standard VA questionnaires for three age groups: child aged under 4 weeks, child aged 4 weeks to 14 years, and person aged 15 years and above. ${ }^{20}$ They also provided standardized methods of certification, coding, and tabulation of causes of death from VAs according to the International Statistical Classification of Diseases and Related Health Problems, tenth revision (ICD-10). ${ }^{20}$ The development of these standards was the first step. Further research includes optimization of the questionnaires and the refinement of reliable methods for assigning cause of death.

\section{VA: standardization, validation, and recent research}

Survey tools such as the VA are subject to underestimation due to underreporting, underrepresentation, and misclassification. ${ }^{12}$ The VA in particular is also limited by issues of standardization and validation. A standardized VA instrument does not exist (although this is the goal of the 2007 WHO VA standards), and standardized methods of VA administration and cause of death assignment are lacking. ${ }^{21}$ Remaining questions regarding VA administration include the ideal amount of interviewer training, optimal timing of the interview in terms of how long after death the VA may be conducted, and the most appropriate primary respondent to the interview. ${ }^{21}$ In previously published VA studies, the training of interviewers has ranged from days to weeks, timing of the interview has ranged from days to up to 5 years after death, and the mother is most frequently the primary respondent. ${ }^{21}$ The best method of cause of death determination is an active area of VA research; the use of computer algorithms to assign a cause of death are being evaluated, ${ }^{21-28}$ but the use of two- or three-physician review has historically been used. In a study conducted in rural Ghana, Edmond et al assessed VA reviewer agreement using the 2002 revised version of the WHO childhood VA. ${ }^{29} \mathrm{~A}$ total of 1251 VAs of either stillbirth or neonatal death were coded by three physician reviewers, and agreement among coders for the four main causes of neonatal death (infection, birth asphyxia, prematurity, and congenital anomaly) was good 
(kappa statistics of $0.72,0.77,0.80$, and 0.63 , respectively). Agreement among reviewers for cause of stillbirth varied, but generally was not as good as those for neonatal deaths. ${ }^{29}$

Validation of the VA tool has also become a more active area of research, and recently a set of over 12,000 VAs (1629 of which are neonatal deaths and 1002 stillbirths) that have accompanying gold standard diagnoses (causes of death determined in health facilities with the help of laboratory, pathology, and medical imaging findings) has been published..$^{30}$ The VA instrument used in this study was based on the 2007 WHO VA standards. ${ }^{30}$ Lozano et al utilized this dataset to attempt validation of the VA tool by comparing single-physician review to gold standard diagnosis. They found that the overall chance-corrected concordance for physician-certified VA against the gold standard cause of death for all age groups was less than $50 \%{ }^{27}$

Lozano et al's results conflict with Edmond et al's study, in which a total of 502 VAs were available for comparison to hospital records. ${ }^{29}$ Sensitivities of the VA to accurately diagnose the various causes of neonatal death were generally higher than those for the various causes of stillbirth (sensitivity $>70 \%$ for all major causes of neonatal death except prematurity). In general, specificity for determining all causes of stillbirth and neonatal death were higher than $90 \%$, except for birth asphyxia (76\%) and infection (87\%). ${ }^{29}$ These sensitivities and specificities, although not for the 2007 version of the WHO VA, can give researchers confidence in the VA's diagnostic accuracy for neonatal death, but there is still a question of its ability to diagnose cause of stillbirth. Overall, however, it should be reiterated that consensus among the global health community on the use of a single VA instrument (including the 2007 WHO VA standards) and the validation and standardization of that specific instrument has yet to be achieved.

\section{Use of VA in a neonatal and perinatal mortality needs assessment for the Loreto Province of Peru: a case study Background}

Educational programs including the American Academy of Pediatrics' Helping Babies Breathe (HBB) and the WHO's Essential Newborn Care (ENC) are being taught all over the world in an effort to decrease rates of neonatal mortality. With the dearth of information from specific locations about the occurrence of neonatal and perinatal mortality, the site-specific causes of neonatal and perinatal mortality, and local risk factors for neonatal and perinatal mortality, it is difficult to know if the educational programs introduced are actually targeted to the needs of the community in which they are taught.

For this reason, a needs assessment was done for nine villages in the Loreto Province, a region in northeast Peru that consists mainly of the Amazon River basin. Peru is one of 68 countries worldwide that accounts for $95 \%$ of maternal and child deaths. ${ }^{31}$ In 2008, the NMR of Peru was reported at 13 per 1000 live births. ${ }^{32}$ In 2005, the perinatal mortality rate (PMR) of Peru, important to consider because of the potential misclassification of neonatal death as stillbirth in developing countries, was reported at 19.8 per 1000 pregnancies. ${ }^{19,33}$ Specifically, the Loreto Province had an NMR of 24.5 per 1000 live births in 2009 and a PMR of 20.4 per 1000 pregnancies in 2005 , as ascertained by the Peruvian Demographic and Health Survey. ${ }^{33,34}$

Villages in the Loreto Province have limited access to healthcare, and it was suspected that the incidence of neonatal and perinatal mortality would be elevated. As vital registration systems do not exist in this rural area, a survey of women in nine villages in the Province was conducted using the 2007 WHO International Standard VA Questionnaire for death of a child under 4 weeks of age.$^{20}$ In addition to providing cause of death estimates for these villages, this tool allowed the burden of and risk factors for neonatal and perinatal death to be determined. With the information gathered during this needs assessment, educational programs targeted to the specific needs of these villages in the Loreto Province will be able to be designed and implemented.

\section{Methods}

\section{Subject eligibility and recruitment}

Subjects were recruited in July and August of 2010 from clinics conducted by Amazon Promise, a United States-based nonprofit organization that has provided medical care in the Loreto province since 1994. All patients were screened and considered eligible for recruitment if they were female, aged 15 years or older, and had been pregnant at least one time in the 5 years prior to interview. Once determined eligible, women were asked to participate in the study and written informed consent was obtained. If the study participant was not able to read or write, verbal informed consent was obtained. The study was approved by the institutional review board at Oregon Health and Science University (Portland, OR).

\section{Study site}

Iquitos, the capitol of the Loreto Province, is accessible only by boat or plane. Iquitos has private and government-run 
clinics and hospitals. Nauta, a town situated on the banks of the Maranon River, is $100 \mathrm{~km}$ south of Iquitos and accessible by a paved road. From Nauta, where basic medical care is available, the nine villages that were visited ranged between a 30-minute and a 4-hour ride in a boat with a small motor.

\section{Interview}

All participants were interviewed by the author (JBW) and a female Spanish interpreter provided by Amazon Promise. Any woman who had experienced a stillbirth or a neonatal death in the previous 5 years was interviewed using the 2007 WHO International Standard VA Questionnaire for death of a child under 4 weeks of age; questions were answered based on experience from the pregnancy that had resulted in stillbirth or neonatal death. For comparison, any woman who had not experienced a stillbirth or a neonatal death in the previous 5 years was interviewed using a questionnaire based on the 2007 WHO VA; questions were answered based on experience from the most recent pregnancy.

\section{Outcome variables}

The number of pregnancies in the 5 years prior to the interview was recorded for each participant. Outcomes for each pregnancy were obtained. Due to the lack of consistent prenatal care and accurate pregnancy dating methods in the region, determination of gestational age at the time of pregnancy loss was reliant on maternal report.

\section{VA}

Three physicians (two neonatal faculty members and one neonatal fellow from Oregon Health and Science University) independently reviewed each completed VA questionnaire. For neonatal deaths and stillbirths occurring after 28 weeks gestation, reviewers were asked to assign a most likely cause of death. Each patient had three separate cause of death assignments, and a cause of death was determined to be agreed upon if two or more reviewers assigned the same cause of death. Agreement between each reviewer pair was assessed (ie, reviewer pairs one-two, one-three, and two-three); overall percent agreement of the reviewers is reported.

\section{Exposure variables}

Several risk factors have been identified from populationbased studies for all-cause neonatal and perinatal death. ${ }^{11}$ Due to the lack of medical records, data for several of these risk factors were not collected. From known risk factors, maternal age, primiparous status, multiple pregnancy, maternal health, preterm or postterm birth, malpresentation, maternal fever during labor, and timing of membrane rupture were considered; however, only maternal age, primiparous status, multiple pregnancy, preterm or postterm birth, and malpresentation were evaluated for association because of the reliability and objectivity of the data. Other variables were also included: sex of the infant, prenatal care, home delivery, birth weight of the infant, delivery mode, maternal tetanus vaccination, distance from Nauta, and presence of a skilled birth attendant.

\section{Statistical analysis}

All statistical analyses were conducted in Stata ${ }^{\circledR} 11.0$ (StataCorp LP, College Station, TX). NMRs are expressed as the number of deaths between postnatal days zero and 28 per 1000 live births. PMRs are expressed as the number of deaths between 28 weeks gestation and postnatal day seven per 1000 pregnancies. NMRs and PMRs were calculated using a logistic generalized estimating equation model assigning the mother as the clustering variable. The sample NMR and PMR were compared to accepted values for Peru and the Loreto Province using linear contrast. VA reviewer agreement was assessed using percent agreement. The twosided Fisher's exact test was used to evaluate the association between exposure variables and neonatal or perinatal death. Exact logistic regression was performed to explore whether more than one of the variables found to be significant in the univariate analyses could be significantly associated with neonatal or perinatal death. The significance level for all tests was set at 0.05 .

\section{Results}

One-hundred and thirty-two women met inclusion criteria; 130 consented and were interviewed (two women declined interview). Data were collected on 263 pregnancies from the 5 years prior to interview (July 2005 to July 2010). Three of the pregnancies were current at the time of interview; therefore, final outcomes were known for 260 pregnancies. The sample included three sets of twins. Two twin pregnancies resulted in stillbirth for both twins, while the other resulted in one live birth and one stillbirth. In order to minimize bias, each twin pregnancy was treated as one pregnancy and one stillbirth. Outcomes of all pregnancies are presented in Table 1. Seven neonatal deaths, all occurring within the first 7 days of postnatal life, were identified from 229 live births (sum of living, neonatal death, infant death, and childhood death). Six stillbirths, all occurring after 28 weeks gestation, were identified from 
Table I Outcomes data for each pregnancy during the 5 years prior to interview $(n=130)$

\begin{tabular}{lll}
\hline Outcome & Definition & Number \\
\hline Currently pregnant & Current pregnancy, any gestational age & 3 \\
Miscarriage & Loss of a pregnancy between conception and 20 weeks gestation & 25 \\
Stillbirth & Loss of a pregnancy between 20 weeks gestation and delivery & 6 \\
Neonatal death & Death of a newborn between postnatal days 0 and 28 & 7 \\
Perinatal death & Death of a fetus/newborn between the 28th week of gestation and postnatal day 7 & 13 \\
Infant death & Death of an infant between I-I2 months of age & 8 \\
Childhood death & Death of a child between I-5 years of age & 3 \\
Currently living & Currently alive, any age & 211 \\
Total pregnancies & & 263 \\
\hline
\end{tabular}

260 completed pregnancies. Therefore, 13 perinatal deaths were identified from 260 pregnancies.

Using the generalized estimating equation model, the sample NMR was found to be 31.4 per 1000 live births $(95 \%$ confidence interval 15.6-62.3). In comparing the sample NMR to the accepted NMRs for both Peru (13 per 1000 live births) and the Loreto Province (24.5 per 1000 live births), the sample NMR is significantly higher than that reported for Peru $(P=0.014)$, but is not significantly different than that reported for the Loreto Province $(P=0.483)$. Using the same model, the sample PMR was found to be 49.7 per 1000 pregnancies (95\% confidence interval 28.5-85.3). In comparing the sample PMR to the accepted PMRs for both Peru (19.8 per 1000 pregnancies) and the Loreto Province (20.4 per 1000 pregnancies), the sample PMR was significantly higher than those reported for both Peru $(P=0.001)$ and the Loreto Province $(P=0.002)$.

VA (for stillbirth or neonatal death) or a detailed questionnaire based on the VA (for live birth) was completed for each of the 130 interviewed women. The one exception was one woman who had experienced two stillbirths; VAs were completed for both of her stillbirths. General characteristics for these 131 pregnancies are detailed in Table 2 .

VA-identified cause of death assignments for neonatal deaths and stillbirths are shown in Table 3. Neonatal death and stillbirth were analyzed separately as to determine efficiency of the VA at determining cause of death between these two comparison groups. Percent agreement among reviewers for neonatal cause of death was $90.5 \%$ (95\% confidence interval 69.6\%-98.8\%). For the neonatal deaths, infection was determined as the cause of death in three of the seven (43\%), asphyxia in two of the seven (29\%), and preterm delivery and congenital malformations each in one of the seven (each 14\%). Percent agreement among reviewers for cause of stillbirth was $38.9 \%$ (95\% confidence interval $17.3 \%-64.3 \%$ ).

Results of the univariate analyses for risk factors are listed in Table 4. Pregnancy with twins $(P=0.001)$,

Table 2 Characteristics of each pregnancy obtained from detailed interview

\begin{tabular}{|c|c|c|}
\hline Characteristic & $\begin{array}{l}\text { Number of pregnancies } \\
(n=|3| \text { unless specified) }\end{array}$ & $\begin{array}{l}\text { Percent } \\
\text { of pregnancies }\end{array}$ \\
\hline Maternal age $<18$ or $>35$ & 34 & $26 \%$ \\
\hline Primiparous status & 43 & $32.8 \%$ \\
\hline Singleton pregnancy & 128 & $97.7 \%$ \\
\hline Delivery at term (37-42 weeks) & $116 / 128^{a}$ & $90.6 \%$ \\
\hline Malpresentation & 2 & $1.5 \%$ \\
\hline Male sex of infant & 66 & $50.4 \%$ \\
\hline Prenatal care (at least one visit) & 68 & $51.9 \%$ \\
\hline Delivery at home & 112 & $85.5 \%$ \\
\hline Birth weight average for gestation & $86 / 126^{b}$ & $68.3 \%$ \\
\hline Delivery by cesarean section & 4 & $3.1 \%$ \\
\hline Maternal tetanus vaccination & $58 / 65^{c}$ & $89.2 \%$ \\
\hline Distance from Nauta $>2$ hours by boat & 56 & $42.7 \%$ \\
\hline \multicolumn{3}{|l|}{ Birth attendant } \\
\hline Traditional birth attendant & 73 & $55.7 \%$ \\
\hline Family member/alone & 40 & $30.5 \%$ \\
\hline Formally trained health professional & 18 & $13.7 \%$ \\
\hline
\end{tabular}

Notes: ${ }^{\mathrm{a}}$ Three women unsure of gestation; ' ${ }^{\mathrm{b}}$ ive women unsure of size/birth weight of baby; ' ${ }^{\mathrm{d}}$ ata not collected for 66 pregnancies. 
Table 3 Verbal autopsy results for cause of death

\begin{tabular}{|c|c|c|c|}
\hline Patient $^{\mathrm{a}, \mathrm{b}}$ & Reviewer I & Reviewer 2 & Reviewer 3 \\
\hline NI & Infection & Tetanus & Tetanus \\
\hline N2 & Asphyxia & Asphyxia & Asphyxia \\
\hline N3 & Asphyxia & Asphyxia & Asphyxia \\
\hline N4 & Infection & Infection & Infection \\
\hline N5 & Congenital malformation & Airway malformation & Congenital malformation \\
\hline N6 & Bowel obstruction & Infection & Infection \\
\hline N7 & Preterm delivery & Preterm delivery & Preterm delivery \\
\hline SI & Asphyxia & Hydrops & Asphyxia \\
\hline S2 & Asphyxia/preterm & Chorioamnionitis & Asphyxia \\
\hline S3 & Asphyxia & Asphyxia & Asphyxia \\
\hline S4 & Asphyxia/cord prolapsed & Unknown & Asphyxia \\
\hline S5 & Asphyxia/preterm & Chorioamnionitis & Asphyxia \\
\hline S6 & Multiple anomalies & Chorioamnionitis & Asphyxia \\
\hline
\end{tabular}

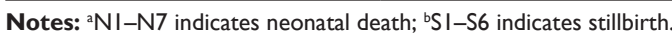

preterm delivery $(P=0.003)$, and delivery by cesarean section $(P=0.049)$ were significantly associated with neonatal or perinatal death. Presence of a doctor at delivery $(P=0.061)$ approached statistical significance.

A sensitivity analysis was performed to ensure that clustering from the one mother who experienced two stillbirths did not alter results of the risk factor analysis. When only one stillbirth was counted for this mother (bringing the total number of perinatal deaths to twelve), results of the risk factor analysis were not substantially different.

Based on the results from exact logistic regression, no single model included more than one significant variable. Of the exposure variables of pregnancy with twins, preterm delivery, and delivery by cesarean section, preterm delivery was the only variable to remain in the exact logistic regression model.

\section{Discussion}

Using direct interview techniques with women who had been pregnant in the 5 years prior to the interview, a needs assessment on neonatal and perinatal death for this area of the Loreto Province was successfully conducted. The NMR and PMR for these villages were both substantial and higher than those currently reported for the country of Peru and the entire Loreto Province, which are based on the Peruvian government's Demographic and Health Survey. The two dominant causes of neonatal death were attributed to infection (43\%) and asphyxia (29\%). VA reviewer agreement was high for cause of neonatal death, but marginal for cause of stillbirth. Pregnancy with twins, preterm delivery, and delivery by cesarean section were significantly associated with neonatal or perinatal death.

Results from the VA cause of death assignments allowed the percentages of cause-specific mortality in the Loreto Province sample to be determined. Comparison with published percentages for both the world (based on statistical modeling) ${ }^{5}$ and Peru (based on $50 \%-74 \%$ vital registration coverage) $)^{32,35}$ is demonstrated in Figure 2. Although the comparison is limited by the small size of the

Table 4 Associations of risk factors to neonatal and perinatal death

\begin{tabular}{|c|c|c|c|}
\hline Risk factor for neonatal or perinatal death & Deaths with risk factor (\%) & Deaths without risk factor (\%) & $P$ value ${ }^{a}$ \\
\hline Maternal age $<18$ or $>35$ & $2 / 34(5.9)$ & II/97 (II.3) & 0.513 \\
\hline Primiparous status & $4 / 44(9.1)$ & $9 / 87(10.3)$ & 1.000 \\
\hline Twin gestation pregnancy & $3 / 3(100.0)$ & $10 / 128(7.8)$ & 0.001 \\
\hline Preterm delivery $(<37 \text { weeks })^{b}$ & $5 / 12(4 I .7)$ & $8 / 116(6.9)$ & 0.003 \\
\hline Nonvertex presentation & $\mathrm{I} / 2(50.0)$ & $12 / 129(9.3)$ & 0.189 \\
\hline Male sex & $8 / 66(12.1)$ & $5 / 65(7.7)$ & 0.561 \\
\hline No prenatal care & $4 / 63(6.3)$ & $9 / 68(13.2)$ & 0.247 \\
\hline Delivery in a medical facility & $4 / 19(21.1)$ & $9 / 112(8.0)$ & 0.096 \\
\hline Birth weight large/small for gestation ${ }^{c}$ & $3 / 40(7.5)$ & $8 / 86(9.3)$ & 1.000 \\
\hline Cesarean section delivery & $2 / 4(50.0)$ & I I/I 27 (8.7) & 0.049 \\
\hline Mother without tetanus vaccination ${ }^{d}$ & $\mathrm{I} / 7(\mathrm{I} 4.3)$ & $12 / 58(20.7)$ & 1.000 \\
\hline Distance from Nauta $>2$ hours by boat & $6 / 56(10.7)$ & $7 / 75(9.3)$ & 1.000 \\
\hline Presence of a doctor at delivery & $4 / 18(22.2)$ & $9 / 113(8.0)$ & 0.061 \\
\hline
\end{tabular}

Notes: aFisher's exact test, two-sided; 'bhree women unsure of gestation at delivery; 'five unknown size, two deaths; ${ }^{\mathrm{d} d a t a}$ not collected for 66 pregnancies. 


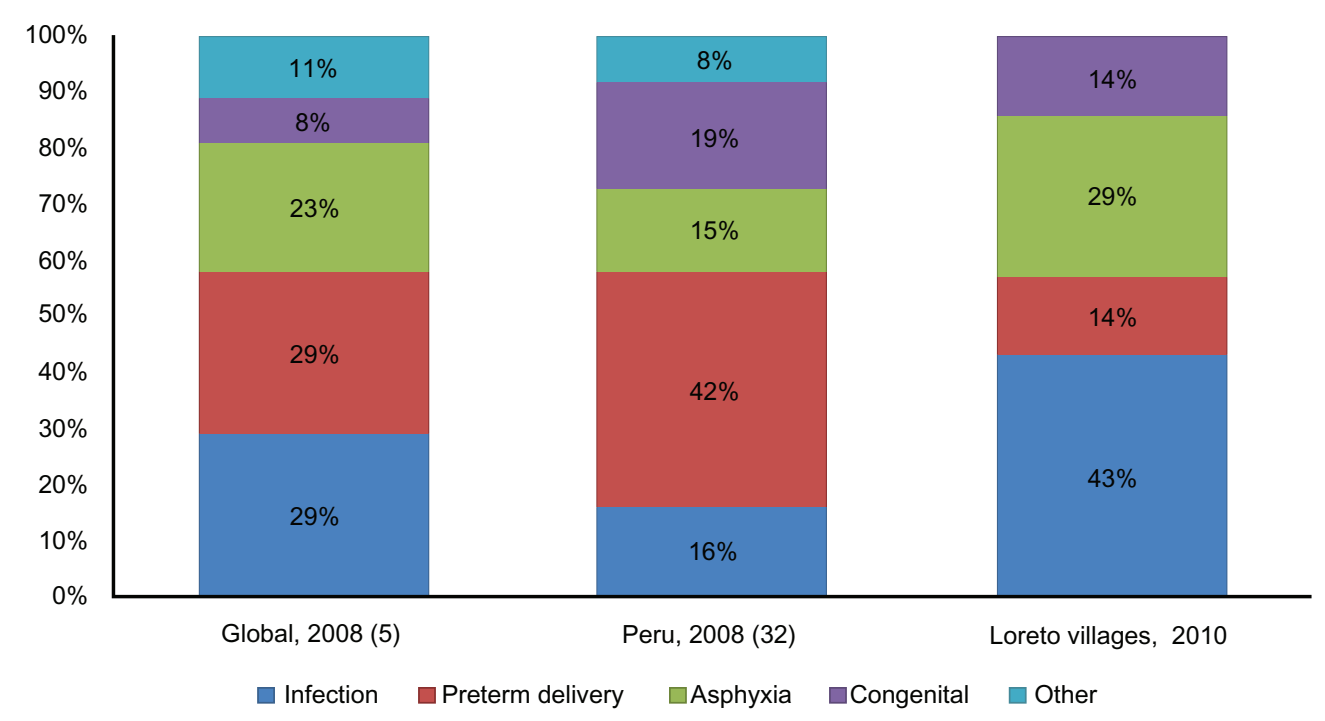

Figure 2 Comparison of neonatal causes of death.

Loreto Province sample, it appears that the Loreto results more closely resemble global than Peruvian government estimates. This discrepancy illustrates differences that can occur in cause-specific mortality estimates due to differences in survey methods. It is possible that the vital registration coverage on which the Peru statistics are based were heavily biased by larger cities with more advanced medical care. This needs assessment was conducted to acquire a more specific understanding of factors contributing to neonatal and perinatal mortality in this province.

A variety of methods are used in conducting needs assessments, including review of available information, use of consultants or experts, and collection of new information. ${ }^{36}$ Due to the remote locations of the many areas with the highest NMRs and PMRs, collection of new information is likely to yield the most valid and reliable information. In the current approach, a survey of patients was utilized to collect new information in order to guide future intervention and curriculum development.

As discussed before, survey tools are subject to underestimation, with the VA also being limited by issues of standardization and validation. ${ }^{12}$ In terms of reviewer agreement for the VA, Edmond et al's results from use of a threephysician review are consistent with the current findings in that better reviewer agreement was seen for neonatal death than for stillbirth. In terms of the diagnostic accuracy of the VA, although not for the particular VA used in the current study, the sensitivities and specificities of the 2002 VA to accurately diagnose the various causes of neonatal death and stillbirth in Edmond et al's study instill confidence in the 2007 WHO VA's ability to diagnose neonatal death, but there is still a question of its ability to diagnose cause of stillbirth. Unfortunately, a large trial such as Edmond et al's study that utilizes the 2007 WHO VA has yet to be conducted, so sensitivities and specificities of the 2007 WHO VA to accurately diagnose the various causes of neonatal death and stillbirth are not available.

The current study has several limitations, the first being its sample size. While conclusions were able to be made about the population studied using this limited number of stillbirths and neonatal deaths, these findings cannot be used to make general statements about other larger populations. Only women who were registered to be seen in clinic were included and could not be compared to those who were not seen in clinic. To increase the sample size, interviews were conducted up to 5 years after the event of death, making recall bias a possibility. As stated earlier, the amount of time for which the VA tool is valid after the occurrence of a stillbirth or a neonatal death is currently unknown. Maternal mortality and young maternal age, two known risk factors for neonatal and perinatal mortality, were not fully addressed since only mothers of the infants who had died were interviewed and women $<15$ years were excluded from participation. The restriction of younger women would result in an underestimation of true NMRs and PMRs. VA cause of death assignments were not able to be validated, as medical records were available for only one of the 13 perinatal deaths. Physicians trained in neonatology assigned causes of death for stillbirths, but because stillbirths are typically due to obstetric complications, better reviewer agreement may have been seen if obstetricians had reviewed this data. However, even in the most advanced medical settings, 
up to $60 \%$ of stillbirths are unexplained. ${ }^{37}$ It is possible that difficulties in explaining the cause of stillbirth are not due to the reviewer, but due to the inherent characteristics of the stillbirths themselves. ${ }^{37}$ Finally, several of the known risk factors for stillbirth or neonatal death were not able to be addressed due to lack of medical records, resources, and concern for subjectivity of responses. In general, the study was quite reliant on maternal memory, knowledge, and, at times, estimation for information on risk factors.

Despite these potential limitations, the study design has multiple strengths. In accordance with current WHO recommendations, the 2007 WHO Standard VA Questionnaire, as well as a three-physician review for cause of death assignment, was used. ${ }^{38}$ The author (JBW), a female physician who specializes in neonatal care, conducted all interviews with the help of a female Spanish interpreter who was from the Loreto Province. In traveling with Amazon Promise, a well-established medical group in these villages, the author and interpreter were viewed as a trustworthy entity, which likely had implications for the fact that only two of the 132 eligible participants declined interview. Data was able to be obtained in a remote area with limited access to medical care, and overall a large number of exposure variables for association with the main outcomes were able to be analyzed. In the assessment of these exposure variables, three were significantly associated with neonatal or perinatal death, one of which (delivery by cesarean section) had not been identified as a risk factor by previous studies.

The results of this needs assessment have allowed four interventions specifically tailored for these communities to be planned. The American Academy of Pediatrics' HBB and the WHO's ENC are two education programs that focus on the care of neonates and have been widely implemented worldwide. ${ }^{39,40} \mathrm{HBB}$ has a focus of ensuring that every baby is breathing, either spontaneously or via bag-mask ventilation, within the first minute of life. ${ }^{39}$ This program is simulationbased and targeted to the midwives and traditional birth attendants that work in low-resource areas. ${ }^{39}$ The implementation of HBB would address neonatal deaths due to birth asphyxia in this population. ENC was designed to improve the health of newborns through basic preventive care including prenatal care of the mother, prevention of intrapartum infection, postnatal promotion of exclusive breast feeding, attention to thermal control, and continued hygienic practices. ${ }^{40}$ The implementation of ENC in the Loreto Province population would address neonatal deaths due to infection.

The finding in these communities that delivery by cesarean section and delivery in the presence of a physician are risk factors for death highlights the need for program implementation that is not often discussed: recognition of and ability to transport to a higher level of care. If these are risk factors for death in these communities, then transport systems are not adequate in these villages. Recognition of and ability to transport are two separate issues. Discussion with the leaders of the villages and provision of a village-wide transport plan for anyone in medical need may address those deaths that are due to a lack of more skilled medical care.

Finally, it is not worth implementing these programs unless progress can be followed over time. It is not feasible to perform an indepth needs assessment such as this on a frequent basis. However, it is possible to educate the traditional birth attendants in each of these villages to record the occurrence and possible cause of death when a perinatal or neonatal death occurs. With the knowledge of how mortality rates and causes of death change over time, the success of implemented programs can be monitored and improved.

Overall, this needs assessment conducted in nine villages in the Loreto Province of Peru revealed a NMR of 31.4 per 1000 live births and a PMR of 49.7 per 1000 pregnancies. Causes of neonatal death were primarily attributed to infection and asphyxia. Pregnancy with twins, preterm delivery, and delivery by cesarean section were significantly associated with neonatal or perinatal death. These results suggest that educational programs such as HBB and ENC would likely benefit the region. It was also discovered that lack of access to care is potentially a major reason for higher rates of neonatal and perinatal mortality, and this might be addressed with village-wide transport plans. Coupled with the ability to follow mortality rates and causes of death over time, the implementation of these interventions has the potential to benefit these communities.

\section{Conclusion}

The worldwide burden of neonatal and perinatal mortality is great, and MDG4 will not be achieved without making strides in decreasing rates of neonatal mortality. The lack of vital registration systems in many of the world's developing countries impedes the ability of researchers to study the occurrence of, causes of, and risk factors for neonatal and perinatal death. VA is a tool that has the ability to provide this information, and progress has been made in recent years on its standardization and validation. This study detailed the use of the 2007 WHO VA for death of a child $<4$ weeks of age in a neonatal and perinatal mortality needs assessment for the Loreto Province of Peru. Using information from this project, an educational intervention that targets the true needs 
of this region can be confidently created. The importance of a needs assessment before program implementation cannot be overstated. In order to truly impact global NMRs, and in turn strive for meeting the goal set forth by MDG4, the specific needs of communities throughout the world must be addressed.

\section{Acknowledgments}

The authors would like to thank Amazon Promise and Patty Webster for their outstanding support and help in coordinating this project; Rosa Enma Aranzabal Durand and Marcia Gomez for their help with translation; and Joseph Gilhooly, JoDee Anderson, and Daniel Morrow for their time spent reviewing verbal autopsies. Most of all, the authors thank all of the mothers who participated in these interviews.

\section{Disclosure}

The authors report no conflicts of interest in this work.

\section{References}

1. Victora CG. Measuring progress towards equitable child survival: where are the epidemiologists? Epidemiology. 2007;18(6):669-672.

2. United Nations General Assembly. United Nations millennium declaration. September 18, 2000. Available from: http://www.un.org/ millennium/declaration/ares552e.pdf. Accessed October 29, 2010.

3. United Nations. The Millennium Development Goals Report 2011. New York, NY: United Nations; 2011.

4. UNICEF. The State of the World's Children 2012: Children in an Urban World. New York, NY: UNICEF; 2012.

5. Lawn JE, Kerber K, Enweronu-Laryea C, Cousens S. 3.6 million neonatal deaths - what is progressing and what is not? Semin Perinatol. 2010;34(6):371-386.

6. Black RE, Cousens S, Johnson HL, et al. Global, regional, and national causes of child mortality in 2008: a systematic analysis. Lancet. 2010; 375(9730):1969-1987.

7. United Nations. The Millennium Development Goals Report 2010. New York, NY: United Nations; 2010.

8. Rajaratnam JK, Marcus JR, Flaxman AD, et al. Neonatal, postneonatal, childhood, and under-5 mortality for 187 countries, 1970-2010: a systematic analysis of progress towards Millennium Development Goal 4. Lancet. 2010;375(9730):1988-2008.

9. Fenn B, Kirkwood BR, Popatia Z, Bradley DJ. Inequities in neonatal survival interventions: evidence from national surveys. Arch Dis Child Fetal Neonatal Ed. 2007;92(5):F361-F366.

10. Moss W, Darmstadt GL, Marsh DR, Black RE, Santosham M. Research priorities for the reduction of perinatal and neonatal morbidity and mortality in developing country communities. J Perinatol. 2002; 22(6):484-495.

11. Lawn JE, Lee AC, Kinney M, et al. Two million intrapartum-related stillbirths and neonatal deaths: where, why, and what can be done? Int J Gynaecol Obstet. 2009;107(Supp1 1):S5-S18.

12. World Health Organization. Neonatal and Perinatal Mortality: Country, Regional, and Global Estimates. Geneva: World Health Organization; 2007.

13. Paul VK. The current state of newborn health in low income countries and the way forward. Semin Fetal Neonatal Med. 2006;11(1):7-14

14. Lawn JE, Cousens S, Zupan J. 4 million neonatal deaths: when? Where? Why? Lancet. 2005;365(9462):891-900.
15. Baqui AH, Darmstadt GL, Williams EK, et al. Rates, timing and causes of neonatal deaths in rural India: implications for neonatal health programmes. Bull World Health Organ. 2006;84(9):706-713.

16. Freeman JV, Christian P, Khatry SK, et al. Evaluation of neonatal verbal autopsy using physician review versus algorithm-based cause-of-death assignment in rural Nepal. Paediatr Perinat Epidemiol. 2005;19(4): 323-331.

17. Martines J, Paul VK, Bhutta ZA, et al. Neonatal survival: a call for action. Lancet. 2005;365(9465):1189-1197.

18. Marsh DR, Sadruddin S, Fikree FF, Krishnan C, Darmstadt GL. Validation of verbal autopsy to determine the cause of 137 neonatal deaths in Karachi, Pakistan. Paediatr Perinat Epidemiol. 2003;17(2):132-142.

19. Carlo WA, Goudar SS, Jehan I, et al. Newborn-care training and perinatal mortality in developing countries. $N$ Engl J Med. 2010;362(7): 614-623.

20. World Health Organization. Verbal Autopsy Standards: Ascertaining and Attributing Cause of Death. Geneva: World Health Organization; 2007:112.

21. Thatte N, Kalter HD, Baqui AH, Williams EM, Darmstadt GL. Ascertaining causes of neonatal deaths using verbal autopsy: current methods and challenges. J Perinatol. 2009;29(3):187-194.

22. James SL, Flaxman AD, Murray CJ. Performance of the tariff method: validation of a simple additive algorithm for analysis of verbal autopsies. Popul Health Metr. 2011;9:31.

23. Murray CJ, James SL, Birnbaum JK, Freeman MK, Lozano R, Lopez AD. Simplified symptom pattern method for verbal autopsy analysis: multisite validation study using clinical diagnostic gold standards. Popul Health Metr. 2011;9:30.

24. Flaxman AD, Vahdatpour A, James SL, Birnbaum JK, Murray CJ. Direct estimation of cause-specific mortality fractions from verbal autopsies: multisite validation study using clinical diagnostic gold standards. Popul Health Metr. 2011;9:35.

25. Flaxman AD, Vahdatpour A, Green S, James SL, Murray CJ. Random forests for verbal autopsy analysis: multisite validation study using clinical diagnostic gold standards. Popul Health Metr. 2011;9:29.

26. Vergnano S, Fottrell E, Osrin D, et al. Adaptation of a probabilistic method (InterVA) of verbal autopsy to improve the interpretation of cause of stillbirth and neonatal death in Malawi, Nepal, and Zimbabwe. Popul Health Metr. 2011;9:48.

27. Lozano R, Lopez AD, Atkinson C, et al. Performance of physiciancertified verbal autopsies: multisite validation study using clinical diagnostic gold standards. Popul Health Metr. 2011;9:32.

28. Lozano R, Freeman MK, James SL, et al. Performance of InterVA for assigning causes of death to verbal autopsies: multisite validation study using clinical diagnostic gold standards. Popul Health Metr. 2011;9:50.

29. Edmond KM, Quigley MA, Zandoh C, et al. Diagnostic accuracy of verbal autopsies in ascertaining the causes of stillbirths and neonatal deaths in rural Ghana. Paediatr Perinat Epidemiol. 2008;22(5):417-429.

30. Murray CJ, Lopez AD, Black R, et al. Population Health Metrics Research Consortium gold standard verbal autopsy validation study: design, implementation, and development of analysis datasets. Popul Health Metr. 2011;9:27.

31. Bhutta ZA, Chopra M, Axelson H, et al. Countdown to 2015 decade report (2000-2010): taking stock of maternal, newborn, and child survival. Lancet. 2010;375(9730):2032-2044.

32. Bryce J, Harris Requejo J; World Health Organization; UNICEF. Countdown to 2015 Decade Report (2000-2010) with Country Profiles: Taking Stock of Maternal, Newborn and Child Survival. Geneva: World Health Organization; 2010.

33. World Health Organization. Peru Country Profile. Geneva: World Health Organization; 2010.

34. National Institute of Statistics and Informatics. Outcome indicators identified in the strategic programs: 2000-1, semester 2010. Demographic and Family Health Survey: ENDES 2010. Lima, July 2010.

35. World Health Organization. Causes of Death 2008: Data Sources and Methods. Geneva: World Health Organization; 2011. 
36. Hughes MT. Step 2: needs assessment of targeted learners. In: Kern DE, Thomas PA, Howard DM, Bass EB, editors. Curriculum Development for Medical Education: A Six-Step Approach. Baltimore: Johns Hopkins University Press; 1998:20-27.

37. Engmann C, Jehan I, Ditekemena J, et al. An alternative strategy for perinatal verbal autopsy coding: single versus multiple coders. Trop Med Int Health. 2011;16(1):18-29.

38. Ye M, Diboulo E, Niamba L, et al. An improved method for physiciancertified verbal autopsy reduces the rate of discrepancy: experiences in the Nouna Health and Demographic Surveillance Site (NHDSS), Burkina Faso. Popul Health Metr. 2011;9:34.
39. AmericanAcademy of Pediatrics. Helpingbabies breathe: the goldenminute May 2010. Available from: http://www.helpingbabiesbreathe.org. Accessed July 2, 2011.

40. Save the Children. Essential newborn care. 2004. Available from: http:// siteresources.worldbank.org/INTPHAAG/Fact\%20Sheets/20559137/ AAGENCSept04.pdf. Accessed July 2, 2011.

\section{Publish your work in this journal}

Research and Reports in Neonatology is an international, peer-reviewed, open access journal publishing original research, reports, editorials, reviews and commentaries on neonatal health. The manuscript management system is completely online and includes a very quick and fair peer-review system. Visit http://www.dovepress.com/testimonials.php to read real quotes from published authors. 